\title{
MIXING BY INTERNAL WAVES
}

\author{
JOSEFINA MONTALBÁN \\ Observatoire de Meudon, 92195 Meudon-Cedex, France \\ EVRY SCHATZMAN \\ Observatoire de Meudon, 92195 Meudon-Cedex, France
}

\section{INTRODUCTION}

That mixing take place in the radiative zone of many stars, is an event that cannot be forgotten when we try to explain observational results as the lithium abundance in the atmosphere of different stars, its dependence on spectral type, age or rotation velocity... During the last years many processes have been proposed as being responsible of this mixing: overshooting, turbulence induced by rotational instabilities, internal waves, etc... We will consider, following the results obtained by Press (1981), the role of this last mechanism in the transport of lithium to the burning level, not as generators of turbulence (in Press, 1981, and García-López and Spruit, 1991, it is shown that turbulence induced by internal waves decays very quickly inside the radiative zone), but as generators of a diffusive process due to non linear dissipative effects.

\section{DIFFUSION COEFFICIENT}

The motions in the convective zone can generate a velocity field which, at the boundary of the convective zone, generates gravity waves which propagate inside the star. Press has estimated the flux of mechanical energy carried in the radiative zone by gravity waves excited at the boundary of the convective zone and has also given an estimate of their radiative damping. For us, the most significative result of Press is the event that due to the radiative damping, the gravity waves generates a second order irreversible motion of fluid elements that can be interpreted as a macroscopic diffusive process which transports chemical elements.

The diffusion coefficient can be expressed as the product of a length by a velocity. The length is the distance reached by a fluid element after an overturn $\omega^{-1}$; the velocity $\mathbf{v}$ is the product of the distance by the circular frequency, $v=l \cdot \omega$. For a gravity wave with a vertical wave number $k_{v}$ and a vertical velocity $u_{v}$, Press gives as an order of magnitude

$$
l=\frac{k_{v} u_{v}^{2}}{\omega^{2}} \frac{D_{t h} k_{v}^{2}}{\omega}
$$

where $D_{t h}$ is the thermal diffusivity, the first factor is the displacement in an overturn and the second is the ratio of the time of an overturn to the time of heat diffusion over a wave length $k_{v}$. It can be considered as a measure of the 
irreversible process, or as fraction of the entropy memory which is lost in the irreversible process. Schatzman (1991), following the works by Press (1981) and García-López and Spruit (1991) determined a diffusion coefficient due to internal waves. In order to obtain it he considered:

- The expression given by Press for the vertical velocity as a function of depth.

- That the horizontal velocity component is generated by the turbulent flow, and the relationship between the vertical and horizontal components of the motion is that corresponding to a sharp change of the Brunt-Väisälä frequency. That last hypothesis is justified by the new model of convective penetration (Zahn, 1991).

- The effects of the number of cells of size $\mathbf{k}$ which contribute to the horizontal displacements generating the wave $\left(\omega, k_{H}\right)$.

- That is possible to describe the effects of the turbulence spectrum by the Kolmogorov spectrum.

- That in the damping term is possible to separate the radius dependence $(F(r))$ from the $k$ dependence.

The result is that the diffusion coefficient decreases very quickly from the bottom of the convective zone, like $\left(r_{c}-r\right)^{-4.6}$. Therefore, the value of the lithium depletion which will arise from the diffusion equation will be very senstive to the variation of the depth of the convective zone. And it will be important to take into account the events which can change this depth: convective penetration, age of the star...

\section{COMPUTATION OF DEPLETION}

The concentration of lithium in the radiative region obeys a diffusion equation where the nuclear reactions are taken into account:

$$
\rho \frac{\partial c}{\partial t}=\frac{\partial}{\partial z}\left(D(z) \rho \frac{\partial c}{\partial z}\right)-K(\rho, T) \rho c
$$

where $\mathrm{z}$ is the geometrical depth, $\rho(z)$ and $T(z)$ are the density and temperature given by the envelope model as a function of $z, K(\rho, T)$ is the nuclear destruction rate taken from Rolfs and Kavanagh (1986), and $D(z)$ is the above diffusion coefficient.

The method used to resolve the diffusion equation is that one used in Baglin et al. (1985), that is, the solution can be approximated by

$$
c(z, t)=c(z) e^{\lambda t}
$$

and with the boundary conditions, diffusion equation leads to an eigenvalue problem. Its solution, $\lambda$, gives us the depletion rate at a given age, for an given model and a given diffusion coefficient. 


\section{MODELS USED IN THE COMPUTING}

The models employed in the computing of the lithium abundance in the Sun have been computed using the new code by P. Morel (1992) which introduces a recent model of convective penetration ( $\mathrm{Zahn}, 1991)$. Opacities are from the Livermore opacity library (1991). The chemical composition is $X=0.706, Z=0.02$. And the value of the classical mixing length parameter is $\alpha=1.9$.

We have determined the lithium depletion rate in the Sun at six different ages, from $0.0 \mathrm{Gyr}$. until $4.75 \mathrm{Gyr}$; and for several values of $\zeta$, the parameter which describes the convective penetration.

\section{RESULTS}

For every $\zeta$ we obtained that lithium depletion rate is a function of time. That is, the Lithium has been burning faster in the early Sun than in the present Sun, in a factor that is almost constant for all the $\zeta$ taken. Then, the lithium abundance at time $t$, may be calculated by

$$
[L i](t)=[L i](t=0) \exp \left(-\int_{0}^{t} \lambda(t) d t\right)
$$

The fitting of the lithium abundance to the observed values will permit us to determinate the parameter $\zeta$. The result $(\zeta=0.3)$ is in good agreement with the value obtained by G. Berthomieu et al. (1992) for the description of solar oscillations.

\section{REFERENCES}

Baglin, A., Morel, P.J., Schatzman E.: 1985, Astron. Astrophys. 149, 309.

Berthomieu, G., Morel, P., Provost, J., Zahn, J-P.: 1992, (To be published in these proccedings).

García-López, R. and Spruit, H., 1991, Astrophys. J., 337, 268.

Press W.H.: 1981, Astrophys. J., 245, 286.

Rolfs, C. and Kavanagh, R.W.: 1986, Nucl. Phys., vol. A455, p. 179.

Schatzman E.: 1991, in Angular Momentum Evolution of Young Stars, S. Catalano and J.R. Stauffer Eds., Kluwer Academic Publishers, p. 223.

Zahn, J-P.: 1992, Astron. Astrophys., 252, 179. 